\title{
Analysis of Trace Forensic Evidence using Raman Microscopy
}

\author{
B. Leimer ${ }^{1}$, R. Garbutt ${ }^{2}$ F. Weesner ${ }^{3}$ and J. Hodkiewicz ${ }^{3}$ \\ ${ }^{1}$ Thermo Electron Corporation, 355 River Oaks Pkwy, San Jose, CA 95134 \\ ${ }^{2}$ Sacramento Laboratory of Forensic Services, 4800 Broadway Ste 200Sacramento, CA 95820 \\ ${ }^{3}$ Thermo Electron Corporation, 5225 Verona Rd, Madison, WI 53711
}

Raman analysis is becoming a powerful tool in the forensic laboratory. A variety of samples commonly found in evidence are readily analyzed by Raman spectroscopy. These samples include drugs, paint chips, fiber specimens, explosive materials, and inks. Bulk samples such as drugs in powder or tablet form are effectively analyzed using by various macro sampling accessories. For trace evidence investigations Raman microscopy has many advantages. Raman microscopy provides distinctive, specific identification non-destructively on specimens, or domains within specimens down to a few microns in size. Little if any sample preparation is required leading to rapid completion of the analysis.

Fiber identification is quickly carried out using Raman microscopy and can often be done more definitively than other techniques. Collection of Raman spectra of fiber specimens is straightforward. Specimens are laid out on glass microscope slides and presented to the instrument. Raman spectra are highly specific. Figures 1 and 2 demonstrate the differentiation that is observed in closely related polyolefin and nylons fibers. Even fibers that are closely related chemically can be confidently identified.

Raman microscopy can look beyond base fiber identification to offer identification of specific fiber dyes. This application offers an alternative to the more time consuming process of physically extracting the dye before identification. Figures 3 depicts an example where Raman microscopy is used to identify the dye in a PET fiber. A reference spectrum of PET was subtracted from the fiber spectrum to yield a residual spectrum of the dye.

Paint specimens are also good candidates for Raman microscopy analysis. The surfaces of the paint chip may be examined, or the specimen can be cleaved to show the inner layers. Raman spectroscopy does not require the sample to be thin so no special tools such asmicrotome are needed to prepare a cross section. Figure 4 shows an example of how dispersive Raman can be used to examine and identify the different layers from a small chip of automobile paint. Raman spectra were obtained from each of the three layers (basecoat, pigment, and styrene clearcoat) by targeting each of the three layers one at a time.

All data in this abstract were collected on a Nicolet Almega dispersive Raman spectrometer using a $785 \mathrm{~nm}$ laser excitation through a 50x microscope objective. Spectral resolutions ranging from 4 to $8 \mathrm{~cm}^{-1}$ using a 0.25 meter spectrograph with CCD detector. Typical acquisition times ranged from 20 to 60 seconds for each spectrum. 


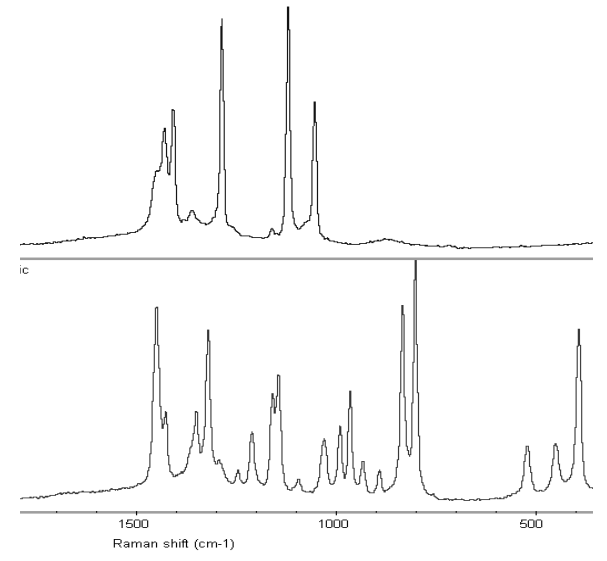

Figure 1. Comparison of polyolefin fibers. Polyethylene (top) and polyproplylene (bottom).

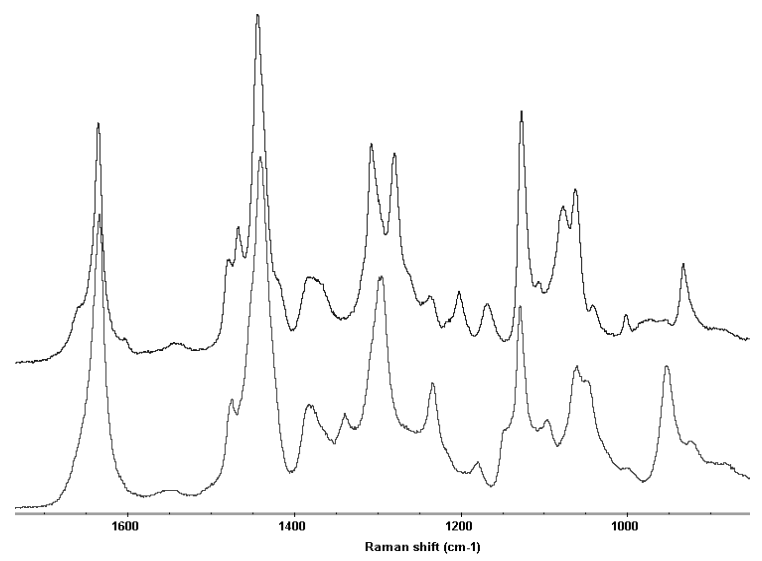

Figure 2. Comparison of two nylon fibers. Nylon 6 (top) and nylon 6,6 (bottom).

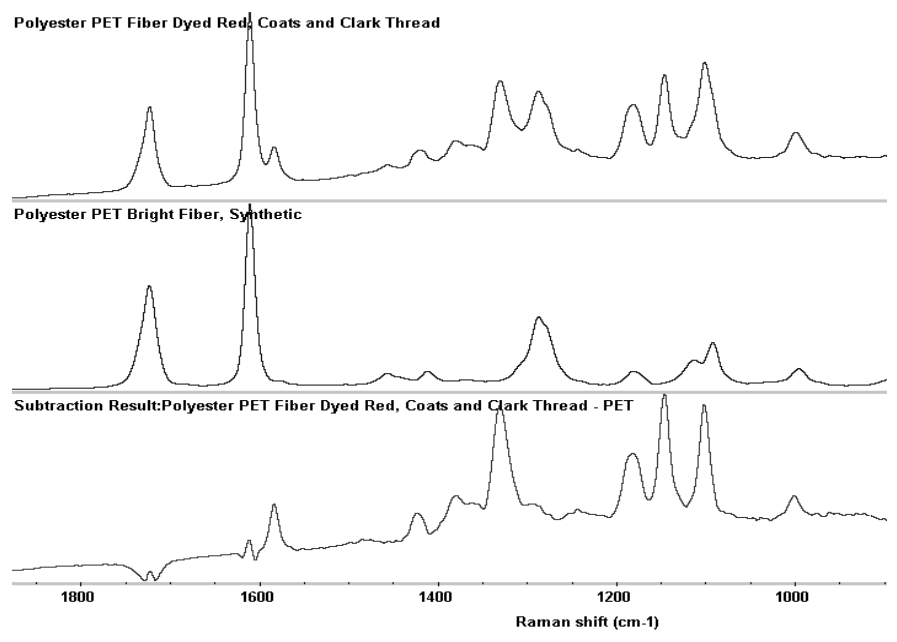

Figure 3. Dyed PET fiber (top), reference fiber (middle), subtraction result (lower). Subtraction result was compared to reference library and identified as nitrophenolazo dye.

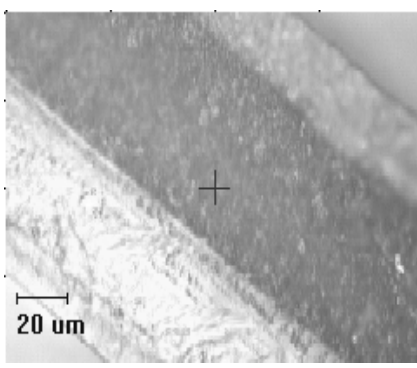

Figure 4. Raman Spectra of three layers of an automobile paint chip.

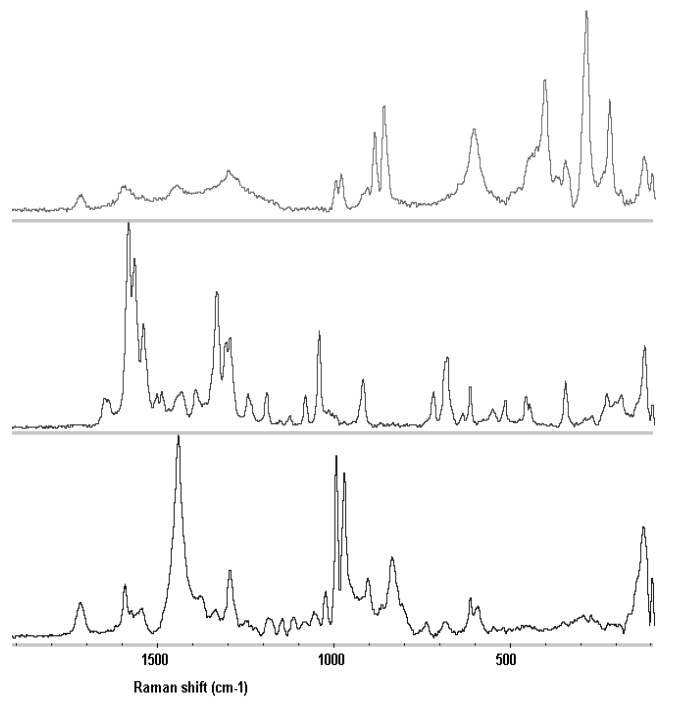

